\title{
Dietary-Induced Variation of Hypoxanthine-Guanine Phosphoribosyl Transferase Activity in Patients with the Lesch-Nyhan Syndrome
}

\author{
William J. Arnold and William N. Kelley \\ From the Division of Rheumatic and Genetic Diseases, Department of \\ Medicine, Duke University Medical Center, Durham, North Carolina 27710
}

\begin{abstract}
A B S T R A C T We have studied three patients with the Lesch-Nyhan syndrome to assess the effect of dietary purines on erythrocyte hypoxanthine-guanine phosphoribosyltransferase (HGPRT) activity. During dietary purine restriction HGPRT activity rose in all three patients; resumption of normal dietary purine intake or the addition of adenine ( $10 \mathrm{mg} / \mathrm{kg}$ per day) to a purinefree diet resulted in a fall in HGPRT activity. These changes in enzyme activity appeared to be due to an activation or inactivation of the mutant enzyme without a change in the half-life or absolute amount of HGPRT enzyme protein.
\end{abstract}

\section{INTRODUCTION}

The X-linked, virtually complete deficiency of hypoxanthine-guanine phosphoribosyltransferase (HGPRT) ${ }^{1}$ activity results in a bizarre neurologic disorder, the Lesch-Nyhan syndrome (1). After the initial description of the enzyme defect, several investigators noted low levels of HGPRT activity in hemolysate from patients with this syndrome $(2,3)$ while most found HGPRT activity undetectable (4-6). More recently we have noted a wide range of HGPRT activity over time in the same patient suggesting that environmental factors might partly account for this discrepancy. Since the deficiency of HGPRT activity in the Lesch-Nyhan syndrome is associated with a normal level of catalytically-incompetent HGPRT protein $(7,8)$, activation of the mutant protein by environmental manipulation

\footnotetext{
Received for publication 30 November 1972 and in revised form 23 January 1973.

${ }^{1}$ Abbreviations used in this paper: HGPRT, hypoxanthineguanine phosphoribosyltransferase; GMP, guanosine monophosphate; ODC, orotidylic decarboxylase; OPRT, orotate phosphoribosyltransferase.
}

represents one possible approach to the therapy of patients with this disease. The present report describes the influence of alterations in dietary purine content on HGPRT activity in erythrocytes from three patients with the Lesch-Nyhan syndrome. The changes observed in HGPRT activity are shown to be due to activation or inactivation of preformed HGPRT protein rather than a change in the absolute amount of the protein.

\section{METHODS}

Three patients with the classical Lesch-Nyhan syndrome were hospitalized on the Clinical Research Unit of Duke University Medical Center and received isocaloric diets which, as specified later, were either free of purines, contained a normal quantity of purines or contained the amount of adenine $(10 \mathrm{mg} / \mathrm{kg}$ per day in four divided doses) present in the normal diet. Allopurinol (150-300 $\mathrm{mg} /$ day) was administered orally to each of the patients during the entire period of study.

HGPRT was assayed in hemolysate by a previously described radiochemical technique using guanine as substrate $(8,9) .\left[8{ }^{14} \mathrm{C}\right]$ guanine $(60 \mathrm{mCi} / \mathrm{mmol})$ was obtained from New England Nuclear Corp., Boston, Mass. Identification of the product of the reaction as guanosine monophosphate (GMP) after incubation of mutant hemolysate with $\left[{ }^{14} \mathrm{C}\right]$ guanine, magnesium, and PP-ribose- $\mathrm{P}$ was confirmed in four different chromatographic systems as described previously (8). Background (150-250 cpm) was defined by the radioactivity migrating with carrier GMP after incubation of a dialysed preparation of hemolysate with a reaction mixture which was complete except for the absence of PPribose-P. Each increment in specific activity of $0.001 \mathrm{nmol} /$ mg per $h$ represented 75 counts above background. Orotate phosphoribosyltransferase (OPRT), and orotidylic decarboxylase (ODC) were assayed as described previously (10, 11).

Density fractionation of intact circulating erythrocytes was also performed by a previously described technique (8). Semiquantitative immunodiffusion using a monospecific rabbit antisera to a homogeneous preparation of normal 

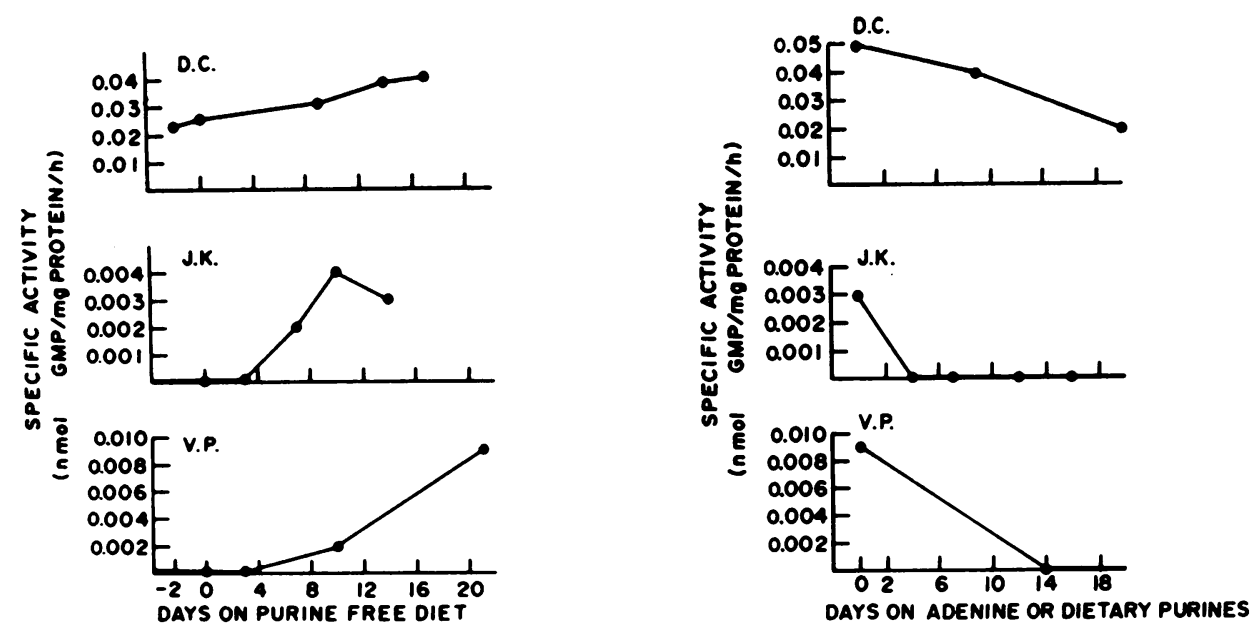

FIGURE 1 The effect of the dietary purines or adenine on erythrocyte HGPRT activity. The panel on the left illustrates the effect of purine restriction on erythrocyte HGPRT activity while the panel on the right illustrates the effect of normal dietary purine content either by the administration of adenine (D. C. and J. K.) or by reinstitution of a diet containing a normal amount of purines (V.P.) on HGPRT activity.

human erythrocyte HGPRT was performed as previously described (8). This technique was standardized by using known quantities of pure human HGPRT. Changes in concentration of $13 \mu \mathrm{g} / \mathrm{ml}$ (representing approximately $10 \%$ of the HGPRT protein present in $1 \mathrm{ml}$ of hemolysate) could be detected (8).

\section{RESULTS}

The effect of a purine-free diet on the level of HGPRT activity in hemolysate from three patients with the Lesch-Nyhan syndrome is illustrated in the left hand panel of Fig. 1. Each patient demonstrated an increase in erythrocyte HGPRT activity beginning from 7 to 14 days after withdrawal of purines from the diet. In two of the three patients the activity was initially undetectable and rose at least three- to ninefold; in the third patient an increase to $54 \%$ above control values was observed.

The right hand panel of Fig. 1 illustrates the reduction in HGPRT activity seen with the addition of adenine to a purine-free diet (patients D. C. and J. K.) or the resumption of a diet with a normal content of purines (patient V. P.). In two of the patients, erythrocyte HGPRT activity became essentially undetectable (J. K. and V. P.) while in the third patient (D. C.) the HGPRT activity decreased by $58 \%$.

The standard error of the mean observed from 10 different determinations of HGPRT activity in a single sample of hemolysate from D. C. and V. P. was $2.9 \%$ and $5.2 \%$ of the mean activity respectively. This, as well as the consistent overall trend in all three patients, suggests that the changes in HGPRT activity observed with dietary manipulation cannot be attributed to assay variability. In all three patients the activity of OPRT and ODC was unchanged during the entire period of s.udy.

The effect of adenine administration on HGPRT activity in circulating erythrocytes fractionated by their density is shown in Fig. 2. HGPRT activity decreased to approximately the same extent in all cell fractions; thus the apparent half-life of HGPRT activity in vivo was unchanged.

The effect of dietary purine restriction on the level of immunoreactive HGPRT protein in each of the three patients is illustrated in Table I. Despite the increase in

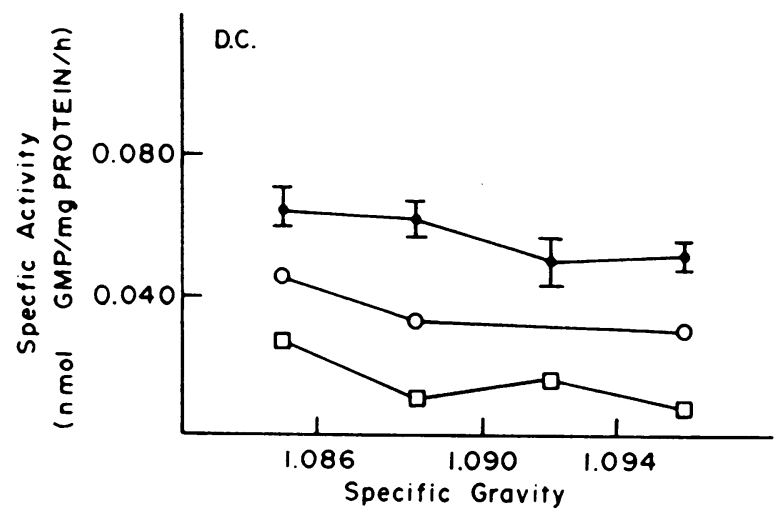

Figure 2 The effect of adenine administration $(10 \mathrm{mg} / \mathrm{kg}$ per day) on HGPRT activity in density-fractionated erythrocytes from patient $\mathrm{D}$. C. Range of two separate determinations during the control period (vertical bars); 9 days after beginning adenine $(0-0) ; 14$ days after beginning adenine ( $\square-\square)$. 
TABLE I

Comparison of HGPRT Activity and Immunoreactive HGPRT Enzyme Protein (CRM) Before and After Purine Restriction in Three Patients with the Lesch-Nyhan Syndrome

\begin{tabular}{llcc}
\hline Patient & \multicolumn{1}{c}{ Diet } & HGPRT activity & $\begin{array}{c}\text { HGPRT protein } \\
\text { (CRM) }\end{array}$ \\
\hline & & $n m o l / m g / h$ & $\mu g / m l$ hemolysate \\
D. C. & Constant purine & 0.022 & 133 \\
& Purine free* & 0.037 & 133 \\
J. K. & Constant purine & $<0.001$ & 130 \\
& Purine fre* & 0.003 & 130 \\
V. P. & Constant purine & $<0.001$ & 133 \\
& Purine free* & 0.003 & 133 \\
\hline
\end{tabular}

* These values were obtained 14-17 days after the initiation of purine restriction.

enzyme activity, the amount of enzyme protein was unchanged.

\section{DISCUSSION}

These studies demonstrate that exogenous influences in the form of dietary purines can alter the phenotypic expression of the basic genetic defect responsible for the Lesch-Nyhan syndrome, i.e., the level of HGPRT activity. The finding that the oral administration of adenine, at roughly the level ordinarily found in the diet, produces essentially the same effect as an unrestricted diet, suggests that the effect of dietary purines can be at least partly attributed to adenine.

The addition of adenine to the diet results in a decrease of HGPRT activity in young as well as old erythrocytes with no change in the apparent half-life of HGPRT. If adenine were reducing HGPRT activity in vivo by repressing the synthesis of HGPRT protein in nucleated erythrocyte precursors one would anticipate a selective decrease of HGPRT activity in the youngest cells. If adenine were reducing HGPRT activity by destabilization of the protein in vivo, this should be reflected in a shortened apparent half-life of HGPRT in circulating erythrocytes during adenine administration. Since neither of these changes were noted, this suggests that the effect of adenine is due, in some manner, to inactivation of HGPRT activity and not due to a change in the rate of synthesis or degradation of the protein itself. This conclusion is further supported by the observation that the level of immunoreactive HGPRT protein remained constant during dietary purine restriction despite a $60-300 \%$ increase in HGPRT activity.

At present the mechanism responsible for this alteration in HGPRT activity is obscure. The changes of HGPRT activity observed in this study are difficult to relate to the apparent activation of mutant HGPRT observed in vitro by Bakay and Nyhan (12) since the activation reported by these investigators required the presence of normal HGPRT protein.

These observations provide one explanation for the variability of HGPRT activity observed with time in a given patient with the classical Lesch-Nyhan syndrome. However, since each of the three patients in this study had quite different maximal levels of HGPRT activity despite similarities in diet and drug ingestion, other factors must contribute to the variability observed between patients. It seems likely that this is a reflection of the genetic heterogeniety previously documented in this disease (13). In addition, this study suggests that dietary purine restriction could be of therapeutic benefit in some patients. Although we did not observe any striking alterations in the clinical course of our patients during purine restriction it is feasible that salutory effects may become evident after more prolonged dietary restriction.

\section{ACKNOWLEDGMENTS}

This research was supported in part by U. S. Public Health Service Grant no. 14362 and United Cerebral Palsy Foundation Grant no. R-247-71 and supported in part by a grant (RR-30) from the General Clinical Research Centers Program of the Division of Research Resourcés, National Institutes of Health.

\section{REFERENCES}

1. Seegmiller, J. E., F. M. Rosenbloom, and W. N. Kelley. 1967. Enzyme defect associated with a sex-linked human neurological disorder and excessive purine synthesis. Science (Wash. D. C.). 155 : 1682.

2. Mizuno, T., M. Segawa, T. Kurumada, H. Maruyama, and J. Onisawa. 1970. Clinical and therapeutic aspects of the Lesch-Nyhan syndrome in Japanese children. Neuropaediatrie. 2 : 38.

3. Sorenson, L. B. 1970. Mechanism of excessive purine biosynthesis in hypoxanthine-guanine phosphoribosyltransferase deficiency. J. Clin. Invest. 49: 968.

4. Kelley, W. N. 1968. Hypoxanthine-guanine phosphoribosyltransferase deficiency in the Lesch-Nyhan syndrome and gout. Fed. Proc. 27 : 1047.

5. Ghadimi, H., C. K. Bhalla, and D. M. Kirchinbaum. 1970. The significance of the deficiency state in LeschNyhan disease. Acta Paediat. Scand. 59: 233.

6. Sperling, O., G. Elion, R. Schmidt, G. Mundel, and A. De Vries. 1971. Purine base incorporation into erythrocyte nucleotides in hypoxanthine-guanine phosphoribosyltransferase deficiency. Biochem. Med. 5: 173.

7. Rubin, C. S., J. Dancis, L. C. Yip, R. C. Nowinski, and M. E. Balis. 1971. Purification of IMP : pyrophosphate phosphoribosyltransferases, catalytically incompetent enzymes in Lesch-Nyhan disease. Proc. Natl. Acad. Sci. U.S. A. $68: 1461$

8. Arnold, W. J., J. C. Meade, and W. N. Kelley. 1972. Hypoxanthine-guanine phosphoribosyltransferase: characteristics of the mutant enzyme in erythrocytes from 
patients with the Lesch-Nyhan syndrome. J. Clin. Invest. $51: 1805$.

9. Kelley, W. N., F. M. Rosenbloom, J. F. Henderson, and J. E. Seegmiller. 1967. A specific enzyme defect in gout associated with overproduction of uric acid. Proc. Natl. Acad. Sci. U.S. A. $57: 1735$.

10. Kelley, W. N., T. D. Beardmore, I. H. Fox, and J. C. Meade. 1971. Effect of allopurinol and oxipurinol on pyrimidine synthesis in cultured human fibroblasts. Biochem. Pharmacol. 20: 1471.

11. Kelley, W. N., and T. D. Beardmore. 1970. Allopurinol :
Alteration in pyrimidine metabolism in man. Science (Wash. D. C.). 169: 388.

12. Bakay, B., and W. L. Nyhan. 1972. Activation of variants of hypoxanthine-guanine phosphoribosyl transferase by normal enzyme. Proc. Natl. Acad. Sci. U. S. A. 69: 2523.

13. Kelley, W. N., and J. C. Meade. 1971. Studies on hypoxanthine-guanine phosphoribosyltransferase in fibroblasts from patients with the Lesch-Nyhan syndrome: Evidence for genetic heterogeneity. J. Biol. Chem. 246: 2953 